\title{
SOBRE LA DISTRIBUCIÓN DEL PINGÜINO REY APTENODYTES PATAGONICUS (AVES: SPHENISCIDAE) EN CHILE
}

\author{
ON THE DISTRIBUTION OF THE KING PENGUIN APTENODYTES \\ PATAGONICUS (AVES: SPHENISCIDAE) IN CHILE
}

Alejandro Kusch ${ }^{1,2} \&$ Manuel Marín ${ }^{1,3}$

Por los últimos 50 años las publicaciones más relevantes (e.g. Goodall et al. 1956, Philippi 1964, Johnson \& Goodall 1965, 1967) han sido utilizadas con pocas modificaciones como la base de los antecedentes sobre la distribución de la avifauna de Chile. Posterior a las publicaciones ya mencionadas, en la región austral de Chile la información sobre distribución de aves se ha basado principalmente en las recopilaciones de Humphrey et al. 1970 y Venegas \& Jory 1979. Sin embargo, en los últimos años se ha descuidado la actualización de información, principalmente en lo que se refiere a posición geográfica, fechas y clases de edad de los individuos registrados para Chile, los que son relevantes para vincularlos con áreas reproductivas o con movimientos migratorios, particularmente si se trata de aves marinas consideradas errantes o poco comunes y que poseen grandes rutas migratorias y áreas de alimentación (Woehler 1992).

Una de éstas es el Pingüino Rey, que debido a la falta de revisiones sistemáticas su distribución ha sido publicada de forma muy general. Este trabajo tiene como objetivo aportar registros que detallan la distribución y estacionalidad del Pingüino Rey en el extremo sur de Chile.

Utilizamos datos publicados en la literatura, especímenes de museo y registros inéditos. Los registros inéditos se obtuvieron de observaciones de los autores, adicionalmente se entrevistó a científicos, guías de turismo y pescadores artesanales que frecuentan los canales patagónicos como parte de su trabajo. Se identificó la edad relativa de los ejemplares observados por los autores o cuando se tuvo acceso a material fotográfico siguiendo una clasificación propuesta para este trabajo (Fig. 1). Las descripciones de Harrison (1983) y Jaramillo (2003) diferencian juveniles o inmaduros de adultos por la falta de pigmentación en el pico y color pálido de la placa del cuello, pero existe un periodo de coloración pálida - amarillento con el pico completamente negro que debería corresponder a una etapa intermedia entre juveniles y adultos, debido a que la coloración del pico es más determinante en relación a la edad de los individuos (Nicolaus et al. 2007). Basados

\footnotetext{
Feather Link, Inc. 1013 Westchester way, Cincinnati, OH 45244, USA.

Casilla 19, Punta Arenas. alekusch@yahoo.com.

Los Angeles County Natural History Museum, Section of Ornithology, 900 Exposition Boulevard, Los Angeles CA 90007, USA. Dirección actual: Casilla 15, Melipilla, Chile. mma95@hotmail.com.
} 


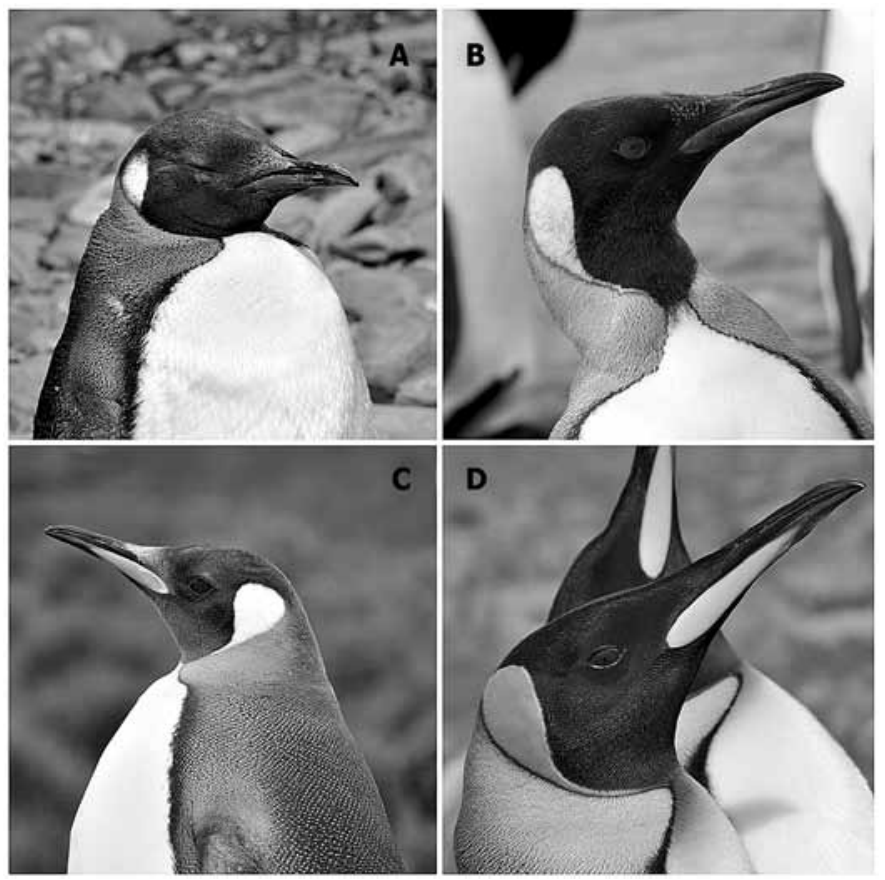

Fig. 1. Diferentes clases de edad del Pingüino Rey. A: juvenil aun con restos de plumón, pico negro y cabeza sin color bien definido. B: juvenil con el pico aún de color negro, las sin placas anaranjadas. C: subadulto con placas del pico ya marcadas pero no los parches del cuello y pecho. D: adulto con placas del pico marcadas y parches de cuello y pecho anaranjados brillantes, contrastando con los colores del cuerpo y cabeza. (Fotografías: M. Marín).

en el trabajo antes mencionado sugerimos que los individuos juveniles corresponden a los que poseen el pico de color negro, además de restos de plumón, ausencia o bajo desarrollo de las placas del cuello color naranja; los individuos subadultos poseen un esbozo de la placa del pico y algo de desarrollo de las placas del cuello y pecho; y los adultos poseen un color definido y bien contrastado de las placas del pico, cuello y pecho.

En general, la distribución del Pingüino Rey (Aptenodytes patagonicus) según Marchant \& Higgins (1990) es de distribución pelágica subantártica y zonas antárticas bajas en el Atlántico sur, océano Índico sur, con individuos errantes llegando al sur de Sur América, África, Australia y Nueva Zelanda. Nidifica en las islas subantárticas entre los $46^{\circ}$ y $55^{\circ}$ $\mathrm{S}$. Colonias de nidificación conocidas se encuentran en las islas Falkland (o Malvinas), Georgias del Sur, una de las islas del norte del grupo South Sandwich, Prince Edward, Marion, Crozet, Kerguelen, Heard, y Macquarie (Murphy 1936, Conroy \& White 1973). Adicionalmente Watson (1975) y Schiavini et al. (2005) insinúan que en el pasado esta especie nidificó en la isla de los Estados (Arg.) y la colonia fue extirpada, posiblemente por loberos, en los alrededores del 1900.

La primera inclusión para Chile en la literatura fue por I.R. Foster para el estrecho de Magallanes en 1781 (sensu Hellmayr \& Conover 1948). Gay (1847) la menciona para Tierra del Fuego y el estrecho de Magallanes. Varios individuos fueron observados en la entrada oriental del estrecho de Magallanes el 5 de diciembre de 1867, pero no se entregó la información sobre la expedición u observador, este antecedente fue mencionado por Humphrey et al. 1970 que citó a Yañez (1948).

Durante la primera mitad del siglo XX, Reynolds (1935) reportó los comentarios de Ken Williams el cual le indico que había observado unos 200 Pingüinos Rey con huevos en octubre de 1930, pero no había podido llegar hasta donde estaban debido a la inclinación del lugar. Lo más probable es que tal reporte esta en error debido a que el Pingüino Rey nidifica en sitios planos a ondulados suaves (ver Conroy \& White 1973). El sitio que describe Williams en Reynolds (1935) correspondería a la Roca Catedral 
donde nidifican Pingüinos de Penacho Amarillo (Clark et al. 1992) y posiblemente Pingüinos Macaroni (M.M obs. pers.). Uno de los nombres alternativos del Pingüino Rey era Pingüino Real (Goodall et al. 1956) y una de las fuentes de confusión en los registros de Pingüino Rey es que el nombre de Pingüino Real, también fue uno de los nombres alternativos que se usaba en la zona austral para el Pingüino de Penacho Amarillo y que ocasionalmente incluían al Pingüino Macaroni (Goodall et al. 1956, De Agostini 2005). En el caso de Reynolds (1935) es muy posible que haya confundido a que especie realmente se referían los informes que recibía. Reynolds (1935) menciona como poco creíble una información recibida por el Capitán Pascualini de Magallanes, sobre la presencia y nidificación del Pingüino Rey lesta información de seguro se refería al Pingüino Real = Pingüino Penacho Amarillo] en las islas Diego Ramírez, este archipiélago es un sitio reconocido por albergar una gran población de Pingüino de Penacho Amarillo y Macaroni (Kirkwood et al. 2007). Murphy (1936) escribió que podría ser un residente en el estrecho de Magallanes y canales fueguinos y mencionó especímenes colectados en el mes de febrero. Para Housse (1945) era una especie frecuente en Patagonia y Chiloé, y Hellmayr \& Conover (1948) mencionan la isla Hornos, seguramente basados en los comentarios de Reynolds, suceso que marcó posteriores antecedentes de distribución del Pingüino Rey. Posteriormente, Philippi (1964) da la especie como casual en Magallanes y que se le ve en la isla hornos (49 $-56^{\circ}$ S). Johanssen (1966, en Humphrey et al. 1970) indica un espécimen muerto y fotografiado en Puerto Nuevo, Bahía Inútil. En el extremo más austral de Chile, se mencionó esta especie como visitante en las islas Diego Ramírez (Schlatter \& Riveros 1987) y por el norte se ha mencionado, sin documentación, que ejemplares errantes han alcanzado hasta isla Guafo $\left(43^{\circ} \mathrm{S}\right)$ (R. Schlatter, com. per., en Venegas $\&$ Sielfeld 1998). Más reciente y poco usual es el reporte de un Pingüino Rey, el dia 30 de noviembre del 2010 en Cartagena (33³2' S, J. L. Brito, com. pers.) siendo este el registro documentado más al norte para la especie por el Pacifico.

Como parte de los resultados, se recopilaron 47 registros de Pingüino Rey, y en 32 ocasiones se pudo obtener la clase de edad de 119 individuos. La mayor parte de los registros corresponden a individuos solitarios o en grupos de 2 a 3 ejemplares, con algunas excepciones. El registro con más individuos fue de 43 a 49 ejemplares en Bahía Inútil durante noviembre del 2010 (C. Silva-Quinta, com. pers.) y es probable de que se encontraran mas pingüinos alimentándose en las cercanías. De los ejemplares revisados, el 87,4\% fueron adultos, 9,2\% subadultos y 3,4\% juveniles. El 57\% de los avistamientos se concentraron en la sección central del Estrecho de Magallanes y pequeñas cantidades en otras zonas de Magallanes. Podría entenderse que esta distribución se debe a un factor de mayor acceso o cercanía para ser detectado. Aunque no se han marcado ejemplares que visiten el estrecho de Magallanes y canales patagónicos, debido a la cercanía con las islas Falkland/Malvinas se trate de ejemplares de dichas poblaciones (Venegas 1994) ya que incluso se han registrado ejemplares que mostraron movimientos de hasta $2000 \mathrm{~km}$, principalmente hacia el mar de Drake (Pütz 2009).

Las últimas observaciones de esta especie en Tierra del Fuego, entre junio del 2011 y febrero del 2012, demostraron que la temporada recién pasada fue la primera donde se observó apareamiento, puesta de huevos y eclosión de 4 crías (A. Kusch obs. pers.), siendo el primer indicio de una probable colonia de A. patagonicus para Chile. Hay que mencionar que el Pingüino Rey tiene un sistema de nidificación poco común, por ejemplo, no construye nido, pone un huevo y con un ciclo (incubación mas crianza) de más de un año, permitiéndole que en un periodo de 3 años solo puede alcanzar como máximo a criar dos polluelos (Watson 1975), lo que implica que para asegurar el asentamiento en un sitio debe conocerse el éxito reproductivo luego de una temporada completa. Actualmente, existen dos fuentes potenciales de origen para los pingüinos Rey en Chile, las cuales son más cercanas al cono sur, las islas Malvinas/Falklands, con una población pequeña entre 250 a 500 parejas (Strange 1992, Woods \& Woods 2006), y en Georgia del Sur, donde se estiman sobre 200000 parejas con una población que está incrementando su número (Burton 2005). El periodo de postura es largo comenzando en noviembre y terminando en abril, y en las islas Falklands/Malvinas, como en otros sitios, existen dos periodos de máxima postura (Van den Hoff et al. 1993, Otley et al. 2007).

Más hacia el sur del continente americano se han encontrado errantes solitarios en la Península Antártica, e.g. islas Rey Jorge, Aitcho, Cuverville 


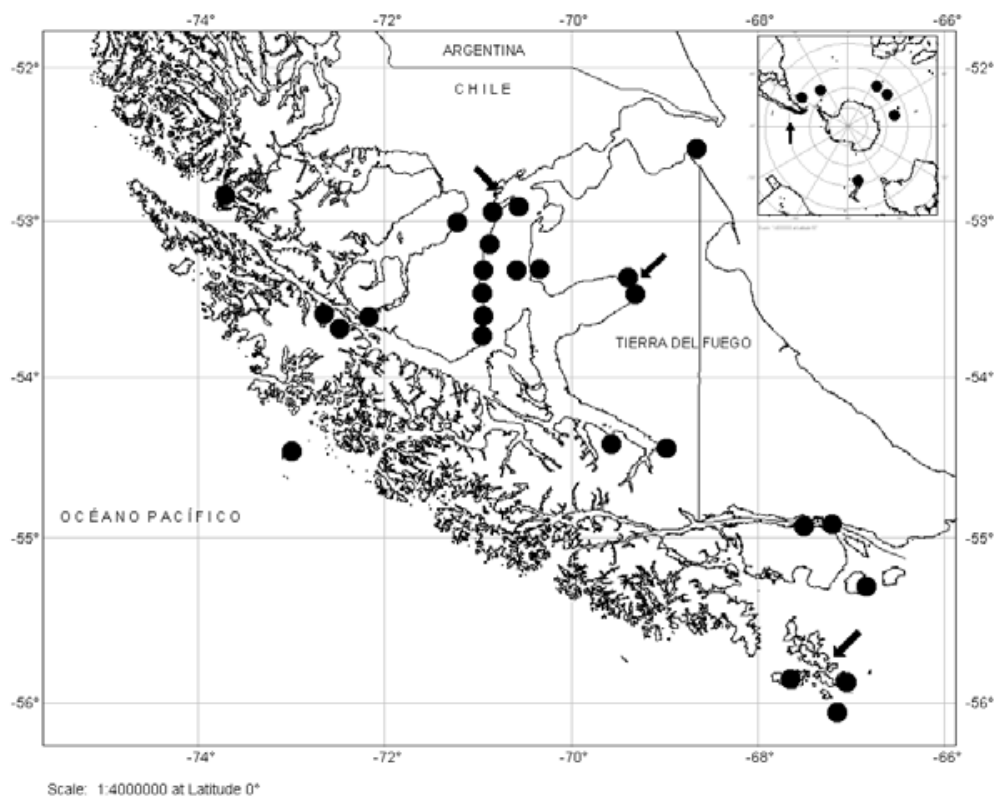

Fig. 2. Distribución geográfica de los registros de Aptenodytes patagonicus en Magallanes. El recuadro indica con una flecha el área de estudio y con puntos las colonias de anidamiento. El mapa principal indica con puntos las localidades de registros y con una flecha los sitios arqueológicos donde se encontraron restos óseos de A. patagonicus.

y Goudier (=Port Locroy $64^{\circ}$ S, M. M. obs. pers.), y hacia el norte por el Atlantico se han observado ejemplares inclusive en la costa de Brasil $\left(23^{\circ}\right.$ a $33^{\circ}$ $\mathrm{S}$, Barquete et al. 2006), lo que muestra el amplio régimen migratorio y por lo que se debería esperar con visitas frecuentes en aguas interiores del estrecho de Magallanes.

Debido a la proporción de adultos registrados $y$ al reciente inicio de una etapa reproductiva es poco probable que el estrecho de Magallanes sea una zona donde los juveniles cambian el plumaje como fue sugerido por Jaramillo (2003), solo uno de los individuos registrados de este trabajo estaba mudando, en Bahía Inútil (octubre del 2002, J. Cárcamo, com. per.).

Basándose en los registros arqueológicos, por varios cientos de años, el Pingüino Rey ha frecuentado las costas del estrecho de Magallanes y Tierra del Fuego, donde se han encontrado restos óseos en sitios del istmo de Brunswick, Bahía Inútil y en el Archipiélago Wollaston (ver fig. 2), en un contexto que debió ser de ítem alimentario oportunístico de los Kaweskar, Selk'nam y Yamanas (Prieto 1988, Lefevré 1993-94, Bahamondez 2004, F. Morello com. per.).

Ambas fuentes de registros, arqueológicos y ornitológicos, indicarían que actualmente las aguas costeras del extremo sur de Sudamérica son parte de la distribución natural para el Pingüino Rey. Anteriormente, Venegas \& Jory (1979) indican que es un visitante accidental y se trata de errantes provenientes de las Malvinas. Luego, Venegas (1994), mencionó que es un visitante de primavera en el estrecho de Magallanes y el $61 \%$ de los registros recopilados en este trabajo ocurrieron en noviembre y diciembre (Fig. 3). La actividad reproductiva observada a partir de noviembre del 2011 corroboraría la posibilidad mencionada por Venegas \& Sielfeld (1998) sobre pingüinos en una búsqueda de un sitio de nidificación en Tierra del Fuego.

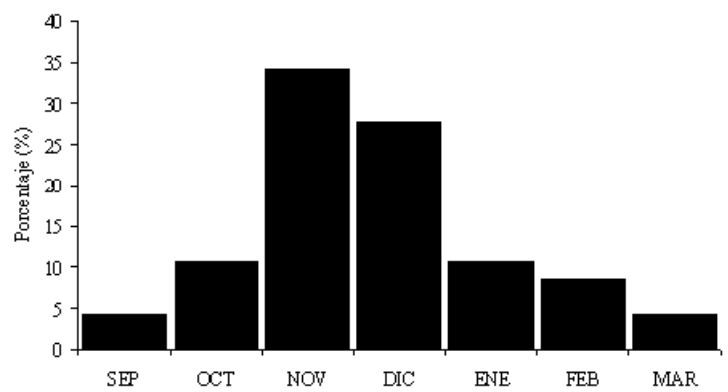

Fig. 3. Distribución mensual de registros $(\mathrm{N}=47)$ de Aptenodytes patagonicus en Magallanes. 
Tabla 1. Registros de Aptenodytes patagonicus principalmente en la zona austral de Chile, Región de Magallanes. Se incluyeron dos observaciones en el sector argentino del canal Beagle, por su cercanía a aguas nacionales.

\begin{tabular}{|c|c|c|c|}
\hline Localidad & Lat.-Lon. & Clases de edad & Fuente \\
\hline $\mathrm{xx}$-sep-2002 isla Lennox & $55^{\circ} 19^{\prime}-66^{\circ} 50^{\prime}$ & 1 adulto & A. Kusch, obs. pers. \\
\hline 07-sep-2007 Pta. Arenas, Est. de Magallanes & $53^{\circ} 03^{\prime}-70^{\circ} 52^{\prime}$ & 1 adulto & R. Matus, com. pers. \\
\hline 10-oct-2002 Pto. Nuevo, Tierra del Fuego & $53^{\circ} 15^{\prime}-69^{\circ} 24^{\prime}$ & 1 adul., 1 sub adul., 1 juvenil & J. Cárcamo, com. pers. \\
\hline 23-oct-2005 Km.35 Sur de Pta. Arenas & $53^{\circ} 17^{\prime}-70^{\circ} 55^{\prime}$ & 1 adulto & La Prensa Austral \\
\hline 25-oct-2010 Pta. Arenas, Est. de Magallanes & $53^{\circ} 03^{\prime}-70^{\circ} 52^{\prime}$ & 2 adultos & $\begin{array}{l}\text { C. Silva-Quinta \& C. de la } \\
\text { Rivera, com. pers. }\end{array}$ \\
\hline 28-oct-2001 Pta. Gruesa, Ba. Ainsworth & $54^{\circ} 24^{\prime}-69^{\circ} 37^{\prime}$ & & E. Devereux, com. pers. \\
\hline xx-oct-2000 Punta Gusano, Isla Navarino & $54^{\circ} 53^{\prime}-67^{\circ} 35^{\prime}$ & 1 adulto & D. Chevallay, com. pers. \\
\hline 02-nov-2010 Ba. Chilota, Tierra del Fuego & $53^{\circ} 18-70^{\circ} 22^{\prime}$ & 1 subadulto & $\begin{array}{l}\text { C. Silva-Quinta \& C. de la } \\
\text { Rivera, com. pers. }\end{array}$ \\
\hline 08-nov-2006 Seno Otway, Magallanes & $52^{\circ} 59^{\prime}-71^{\circ} 14^{\prime}$ & 1 juvenil & R. Matus, com. pers. \\
\hline 09-nov-2005 Cabo Negro, Est. De Magallanes & $52^{\circ} 52^{\prime}-70^{\circ} 47^{\prime}$ & 1 adulto & R. Kusch, com. pers. \\
\hline 09-nov.2010 Ba. Beaufort, I. Emiliano Figueroa & $52^{\circ} 50^{\prime}-73^{\circ} 50^{\prime}$ & 25 a 30 individuos & D. Lleufo, com. pers. \\
\hline 09-nov-2010 $\begin{array}{l}\text { Desembocadura río Marazzi, Tierra } \\
\text { del Fuego }\end{array}$ & $53^{\circ} 27^{\prime}-69^{\circ} 18^{\prime}$ & 5 adultos & $\begin{array}{l}\text { C. Silva-Quinta \& C. de la } \\
\text { Rivera, com. pers. }\end{array}$ \\
\hline 10-nov-2004 Punta Arenas & $53^{\circ} 03^{\prime}-70^{\circ} 52^{\prime}$ & 1 adulto & M. Marín, obs. pers. \\
\hline 11-nov-2005 Punta Arenas & $53^{\circ} 03^{\prime}-70^{\circ} 52^{\prime}$ & 1 adulto & La Prensa Austral \\
\hline 12-nov-2000 Pto. Nuevo, Tierra del Fuego & $53^{\circ} 15^{\prime}-69^{\circ} 24^{\prime}$ & 1 subadulto & J. Hornbuckle \\
\hline 16-nov-2008 Río San Pedro, Magallanes & $53^{\circ} 41^{\prime}-70^{\circ} 48^{\prime}$ & 1 juvenil & La Prensa Austral \\
\hline 16-nov-2010 Punta Arenas & $53^{\circ} 03^{\prime}-70^{\circ} 52^{\prime}$ & 1 adulto & El Pingüino Diario \\
\hline 22-nov-2010 Río Marazzi, Tierra del Fuego & $53^{\circ} 27^{\prime}-69^{\circ} 18^{\prime}$ & 43 adultos & $\begin{array}{l}\text { C. Silva-Quinta \& C. de la } \\
\text { Rivera, com. pers. }\end{array}$ \\
\hline 25-nov-2007 Isla Lennox & $55^{\circ} 19^{\prime}-66^{\circ} 50^{\prime}$ & 1 adulto & J. Plana, com. pers. \\
\hline 30-nov-2004 Punta Arenas & $53^{\circ} 03^{\prime}-70^{\circ} 52^{\prime}$ & 1 adulto & La Prensa Austral \\
\hline 30-nov-2010 Cartagena & $33^{\circ} 32^{\prime}-71^{\circ} 36^{\prime}$ & 1 adulto & J. L. Brito, obs.. pers. ${ }^{1}$ \\
\hline xx-nov-1990 Pto. Zenteno, Est. De Magallanes & $52^{\circ} 54^{\prime}-70^{\circ} 52^{\prime}$ & & Y. Alvarez, com. pers. \\
\hline xx-nov-2002 Pto. Nuevo, Tierra del Fuego & $53^{\circ} 15^{\prime}-69^{\circ} 24^{\prime}$ & & R. Donoso, com. pers. \\
\hline xx-nov-2003 Pto. Nuevo, Tierra del Fuego & $53^{\circ} 15^{\prime}-69^{\circ} 24^{\prime}$ & 3 adultos & J. Cárcamo, com. pers. \\
\hline 02-dic-2004 Punta Arenas & $53^{\circ} 03^{\prime}-70^{\circ} 52^{\prime}$ & 2 subadulto y 1 adulto & La Prensa Austral \\
\hline 05-dic-1867 Boca oriental, Est. de Magallanes & $52^{\circ} 28^{\prime}-68^{\circ} 43^{\prime}$ & & Humphrey et al. 1970 \\
\hline 06-dic-2009 Cabo Negro, Est. De Magallanes & $52^{\circ} 52^{\prime}-70^{\circ} 47^{\prime}$ & & La Prensa Austral \\
\hline 10-dic-2009 Isla Noir & $54^{\circ} 28^{\prime}-72^{\circ} 58^{\prime}$ & 1 adulto & M. Marín, obs. pers. \\
\hline 29-dic-2001 I. Magdalena, Est. de Magallanes & $52^{\circ} 55^{\prime}-70^{\circ} 35^{\prime}$ & 1 adulto & CONAF, com. pers. \\
\hline 29-dic-2010 Río Marazzi, Tierra del Fuego & $53^{\circ} 27^{\prime}-69^{\circ} 18^{\prime}$ & 29 adultos, 3 subadultos & A. Kusch, obs. pers. \\
\hline 31-dic-2009 Punta Arenas & $53^{\circ} 03^{\prime}-70^{\circ} 52^{\prime}$ & 1 adulto & Y. Alvarez, com. pers. \\
\hline xx-dic-1994 Seno Nevado & $53^{\circ} 35^{\prime}-72^{\circ} 40^{\prime}$ & & rez, com. pers. \\
\hline xx-dic-1997 Caleta María, Tierra del Fuego & $54^{\circ} 26^{\prime}-68^{\circ} 59^{\prime}$ & 2 adultos & D. Chevallay, com. pers. \\
\hline xx-dic-2004 Pto. Nuevo, Tierra del Fuego & $53^{\circ} 15^{\prime}-69^{\circ} 24^{\prime}$ & & R. Donoso, com. pers. \\
\hline xx-dic-2006 Pto. Nuevo, Tierra del Fuego & $53^{\circ} 15^{\prime}-69^{\circ} 24^{\prime}$ & & R. Donoso, com. pers. \\
\hline xx-dic-2004 Est. de Magallanes & $53^{\circ} 18^{\prime}-70^{\circ} 39^{\prime}$ & & R. Donoso, com. pers. \\
\hline xx-dic-2009 Seno Ballena & $53^{\circ} 43^{\prime}-72^{\circ} 37^{\prime}$ & 2 sub adul. y 1 juvenil & J. Acevedo, com. pers. \\
\hline 11-ene-1969 I. Magdalena, Est. de Magallanes & $52^{\circ} 55^{\prime}-70^{\circ} 35^{\prime}$ & & Hauff 1972 \\
\hline 26-ene-1985 I. Hermite, Ar. Wollaston & $55^{\circ} 50^{\prime}-67^{\circ} 23^{\prime}$ & & Clark et al. 1992 \\
\hline 30-ene-2004 I. Martillo (Arg.), Canal Beagle & $54^{\circ} 54^{\prime}-70^{\circ} 35^{\prime}$ & 1 sub adulto & Mazar Barnett et al. 2005 \\
\hline xx-ene-2000 Ba. Parry, Tierra del Fuego & & 2 adultos & E. Almonacid, com. pers. \\
\hline xx-ene-2007 Puerto Williams, Isla Navarino & $54^{\circ} 53^{\prime}-67^{\circ} 35^{\prime}$ & 1 adulto & D. Chevalay, com. pers. \\
\hline 03-feb-2007 I. Martillo (Arg.), Canal Beagle & $54^{\circ} 54^{\prime}-70^{\circ} 35^{\prime}$ & 1 adulto & M. Marín, obs. pers. \\
\hline 03-feb-2009 I. Herschel, Ar. Wollaston & $55^{\circ} 52^{\prime}-67^{\circ} 07^{\prime}$ & 1 juvenil & R. Ulph, obs. per. ${ }^{2}$ \\
\hline 07-feb-2010 Ba. Chilota, Magallanes & $53^{\circ} 17^{\prime}-70^{\circ} 24^{\prime}$ & 2 adultos & La Prensa Austral \\
\hline 22-feb-2003 Ca. 2 km S del Cabo de Hornos & $56^{\circ} 00^{\prime}-67^{\circ} 16^{\prime}$ & 1 adulto & M. Marín, obs. pers. \\
\hline 03-mar-1979 Bahía Buena & $53^{\circ} 32^{\prime}-70^{\circ} 56^{\prime}$ & 1 subadulto & CZIP \\
\hline xx-mar-2003 río Batchelor & $53^{\circ} 41^{\prime}-71^{\circ} 55^{\prime}$ & 1 adulto & M. Oyarzún, com. pers. \\
\hline
\end{tabular}

1 Registro con fotografía publicado en www.avesdechile.cl. Página de la Unión de Ornitólogos de Chile.

2 Registro con fotografía publicado en La Chiricoca N 10 , Marzo de 2010. Red de Observadores de Aves de Chile. 


\section{AGRADECIMIENTOS}

Los autores agradecen especialmente a Jorge Acevedo, Eduardo Almonacid, Yayo Alvarado, Jaime Cárcamo, Denis Chevallay, Evelyn Devereux, Roberto Donoso, Cristofer de la Rivera, Ricardo Kusch, David Lleufo, Ricardo Matus, Jordi Plana, Alfredo Prieto, Doña Miguelina Oyarzún, Carlos Silva-Quintas y Guardaparques de Isla Magdalena por compartir sus registros y observaciones. También agradecen a los revisores por mejorar esta nota.

\section{LITERATURA CITADA}

Bahamondes, M. 2004. Las aves en la cultura Selk'nam: estudio del registro arquefaunístico de cuatro sitios de bahía Inútil (tierra del Fuego). Magallania, 32:163-189

Barquete, V., L. Bugoni, R.P. Silva-Filho \& A.C. Adornes 2006. Review of records and notes on King Penguin (Aptenodytes patagonicus) and Rockhopper Penguin (Eudyptes chrysocome) in Brazil. Hornero, 21:45-48

Burton, R. 2005. South Georgia. The Government of South Georgia and the South Sandwich Islands.

Clark, G. S., A. Cowan, P. Harrison \& W.R.P. Bourne 1992. Notes on the seabirds of the Cape Horn Islands. Notornis, 39:133-144

Conroy, J. W. H. \& M. G. White 1973. The breeding status of the King Penguin (Aptenodytes patagonicus). British Antarctic Survey Bulletin, 32:31-40

De Agostini 2005. 30 años en Tierra del Fuego. Ediciones El Elefante Blanco, Bs. Aires, Argentina. Edición original de 1956.

Gay, C. 1847. Historia física y política de Chile. Zoología, Tomo 1, Museo de Historia Natural de Santiago, Chile.

Hauff, J. 1972. A King penguin in the Magellan strait. Bulletin of British Omithological Club, 92(5): 117

Harrison, P. 1983. Seabirds an identification guide. Houghton Mifflin Company, Boston.

Hellmayr, C. E. \& B. Conover 1948. Catalogue of birds of the Americas. Zoological series, Field Museum of Natural History. Vol 13, Part 1, No 2.
Housse, R. 1945. Las aves de Chile en su clasificación moderna, su vida y costumbres. Ediciones de la Universidad de Chile, Santiago.

Humphrey, P.S., D. Bridge, P.W. Reynolds \& R.T. Peterson 1970. Birds of Isla Grande (Tierra del Fuego). Smithsonian Institution and University of Kansas Mus. Nat. History, Lawrence, Kansas.

Jaramillo, A. 2003. Birds of Chile. Helm Field Guides, London.

Johnson, A.W., \& J. D. Goodall 1965-67. The birds of Chile and adjacent regions of Argentina, Bolivia and Peru. vol. 1 \& 2. Platt Establecimientos Gráficos S. A., Buenos Aires Argentina.

Kirkwood, R., K. lawton, C. Moreno, J. Valencia, R. Schlatter \& G. Robertson 2007. Estimates of Southern Rockhopper and Macaroni Penguin numbers at the Ildelfonso and Diego Ramirez archipelagos, Chile, using quadrat and distance-sampling techniques. Waterbirds, 30:256-267

Lefevre, C. 1993-94. Las aves en los yacimientos del archipiélago del cabo de Hornos y del seno Grendi. Anales del Instituto de la Patagonia, Ser. Cs. Humanas, 22:123-136

Marchant, S. \& P.J. Higgins 1990. Handbook of Australian, New Zealand and Antarctic birds. vol. 1 Ratites to Petrels. Melbourne Oxford University Press.

Mazar Barnett, J, G.M. Kirwan, \& J. Minns (eds). 2005. The Neotropical Notebook. Cotinga, 23:87-91

Murphy, R.C. 1936. Oceanic birds of South America. vol. 1. The American Museum of Natural History, New York.

Nicolaus, M., C. Le Bohec, P. M. Nolan, M. GauthierClerc, Y. Le Maho, J. Komdeur \& P. Jouventin 2007. Ornamental colors reveal age in the king penguins. Polar Biology, 31: 53-61

Otley, H., A. Clausen, D. Christie, N. Huin \& K. Pütz 2007. Breeding patterns of King penguins on the Falkland islands. Emu, 107:156-164

Philippi, B.R.A. 1964. Catalogo de las aves de Chile con su distribución geográfica. Investigaciones Zoológicas Chilenas, 11:1-179

Prieto, A. 1988. Cazadores-recolectores del itsmo de Brunswick. Anales del Instituto de la Patagonia, Ser. Cs. Sociales, 18:113-131 
Pütz, K. 2009. Das wanderverhalten von juvenilen Königspinguinen im Süd-Atlantik. Abschlussbericht Projekt 19/07. Antarctic Research Trust. $16 \mathrm{pp}$.

Reynolds, P.W. 1935. Notes on the birds of Cape Horn. Ibis, 5:65-101

Schiavini, A. P. Yorio, P. Gandini, A. Raya Rey \& P.D. Boersma 2005. Los pingüinos de las costas Argentinas: estado poblacional y conservación. Hornero, 20:5-23

Schlater, R.P. \& G.M. Riveros 1987. Historia Natural del Archipiélago Diego Ramírez, Chile. Serie Científica INACH, 47:87-112

Strange, I.J. 1992. A field guide to the wildlife of the Falklands islands and South Georgia. Haper Collins Publishers, Frome.

Van den Hoff, J. Kirkwood, R. J. \& Copley, P. B. 1993. Aspects of the breeding cycle of King Penguins Aptenodytes patagonicus at Heard Island. Marine Ornithology, 21:49-55
Venegas, C. 1994. Aves de Magallanes. Ediciones de la Universidad de Magallanes. Punta Arenas. Venegas, C. \& J. Jory 1979. Guía de Campo para las aves de Magallanes. Instituto Patagonia, Punta Arenas.

Venegas, C. \& W. Sielfeld 1998. Catálogo de los vertebrados de la Región de Magallanes y Antártica Chilena. Ediciones de la Universidad de Magallanes. Punta Arenas.

Watson, G.E. 1975. Birds of the Antarctic and sub-antarctic. American Geophysical Union, Washington D.C.

Woods, R. \& A. Woods 2006. Birds and mammals of the Falkland Islands. WildGuides Ltd., Hampshire.

Woehler, E.J. 1992. Records of vagrant penguins from Tasmania. Marine Ornithology, 20:61-73 
A. KUSCH \& M. MARÍN 